\title{
Polyhedral Process Networks
}

\author{
Sven Verdoolaege
}

Katholieke Universiteit Leuven, Belgium

(LIACS, Leiden, Netherlands)

November 20, 2009 


\section{Outline}

(1) Introduction and Motivation

(2) Polyhedral Process Networks

- Polytope Model

- Dataflow Analysis

- Parametric Counting

- Computing Parametric Upper Bounds

(3) Conclusion 


\section{Outline}

(1) Introduction and Motivation

(2) Polyhedral Process Networks

- Polytope Model

- Dataflow Analysis

- Parametric Counting

- Computing Parametric Upper Bounds 


\section{Motivation}

Goal: map sequential programs to parallel hardware (MPSoC)
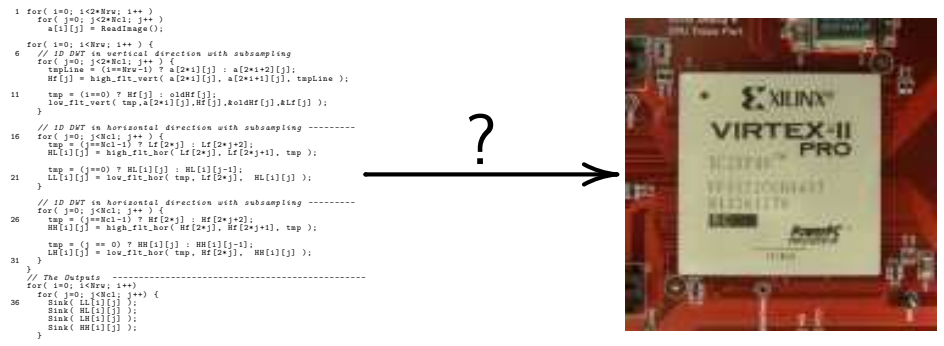


\section{Motivation}

Goal: map sequential programs to parallel hardware (MPSoC)

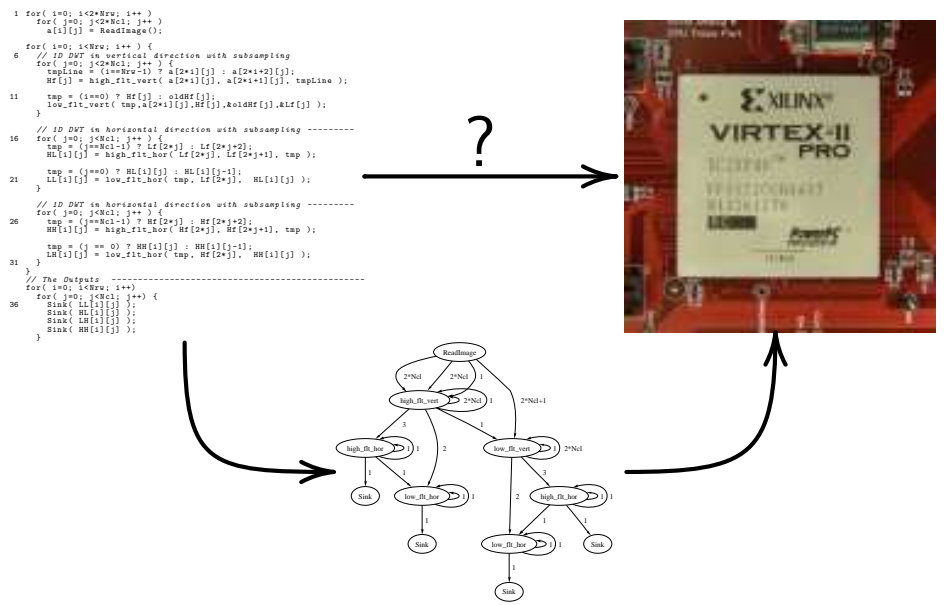

process network 


\section{Kahn Process Networks}

- Parallel specification

$\Rightarrow$ easier to map onto parallel hardware

- Consists of

- independent processes

- communication channels $\Rightarrow$ unbounded FIFOs

- synchronization: blocking read 


\section{Kahn Process Networks}

for $(i=0 ; i<100 ;++i)$ write (fifo, $f(i))$,

for $(i=0 ; i<100 ;++i)$ read (fifo, \&a);

- Parallel specification

$\Rightarrow$ easier to map onto parallel hardware

- Consists of

- independent processes

- communication channels $\Rightarrow$ unbounded FIFOs

- synchronization: blocking read 


\section{Process Networks}

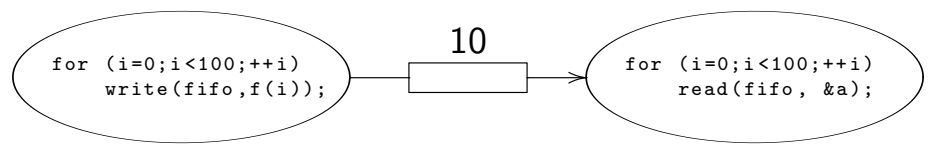

- Parallel specification

$\Rightarrow$ easier to map onto parallel hardware

- Consists of

- independent processes

- communication channels $\Rightarrow \quad$ FIFOs

- synchronization: blocking read + blocking write 


\section{Process Networks}

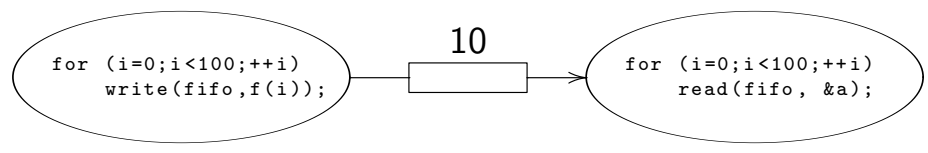

- Parallel specification

$\Rightarrow$ easier to map onto parallel hardware

- Consists of

- independent processes

- communication channels $\Rightarrow \quad$ FIFOs / memories

- synchronization: blocking read + blocking write 


\section{Polyhedral Process Networks}

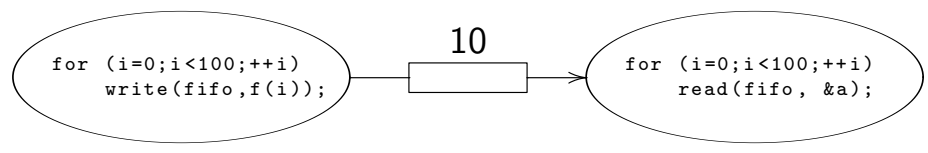

- Parallel specification

$\Rightarrow$ easier to map onto parallel hardware

$\Rightarrow$ automatically derived from sequential program

- Consists of

- independent processes

$\Rightarrow$ one process for each statement

- communication channels

$\Rightarrow \quad$ FIFOs / memories

$\Rightarrow$ obtained from dependence analysis

- synchronization: blocking read + blocking write 


\section{MPSoCs Programming and Mapping}
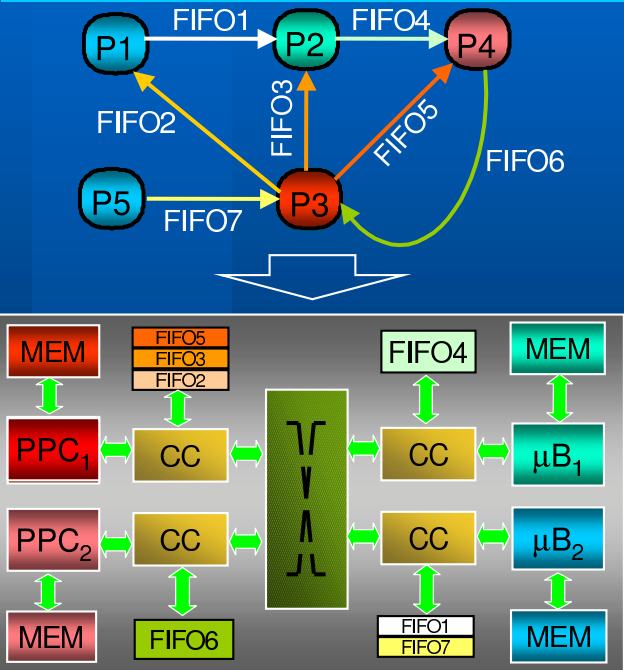

- Programming model: KPN

- Example of a MPSoC platform:

- 2 PowerPC processors

- 2 MicroBlaze processors

- 4 Communication memories (CM)

- 4 Communication controllers (CC)

- Crossbar component

- Mapping:

- The processes are mapped onto the processors using standard GCC compiler.

- The FIFOs are mapped onto the CMs depending on the process mapping.

- CCs take care about the inter processor communication and synchronization 


\section{Outline}

(2) Polyhedral Process Networks

- Polytope Model

- Dataflow Analysis

- Parametric Counting

- Computing Parametric Upper Bounds 


\section{Derivation of Polyhedral Process Networks}

Main difficulty: what are the channels and how big are they ?

- dataflow analysis

Given a read from an array element, what was the last write to that array element? 


\section{Derivation of Polyhedral Process Networks}

Main difficulty: what are the channels and how big are they ?

- dataflow analysis

Given a read from an array element, what was the last write to that array element?

- counting

Given a communication channel, how many tokens are in the channel at any given time? 


\section{Derivation of Polyhedral Process Networks}

Main difficulty: what are the channels and how big are they?

- dataflow analysis

Given a read from an array element, what was the last write to that array element?

- counting

Given a communication channel, how many tokens are in the channel at any given time?

- computing upper bounds

Given a communication channel, what is the maximal number of tokens in the channel during the execution? 


\section{Polytope Model}

$$
\begin{aligned}
\text { for } & (i=1 ; i<=N ;++i) \\
\text { for } & (j=1 ; j<=i ;++j) \\
& a[i][j]=
\end{aligned}
$$

Assumptions on sequential code:

- iterators are integers

- loops with affine bounds

- affine conditions

- affine index expressions 


\section{Polytope Model}

$$
\begin{aligned}
\text { for } & (i=1 ; i<=N ;++i) \\
\text { for } & (j=1 ; j<=i ;++j) \\
& a[i][j]=
\end{aligned}
$$

Iteration domain: $P=\{[i, j] \mid i \geq 1$

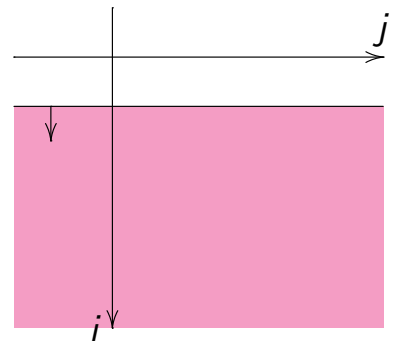

\}

Assumptions on sequential code:

- iterators are integers

- loops with affine bounds

- affine conditions

- affine index expressions 


\section{Polytope Model}

$$
\begin{aligned}
\text { for } & (i=1 ; i<=N ;++i) \\
\text { for } & (j=1 ; j<=i ;++j) \\
& a[i][j]=
\end{aligned}
$$

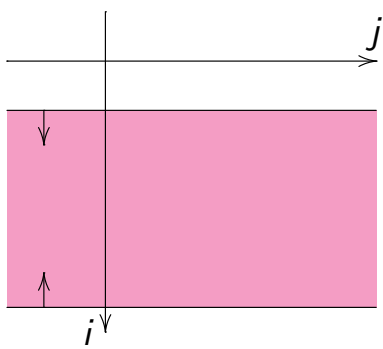

Iteration domain: $P=\{[i, j] \mid i \geq 1 \wedge i \leq N$

Assumptions on sequential code:

- iterators are integers

- loops with affine bounds

- affine conditions

- affine index expressions 


\section{Polytope Model}

$$
\begin{aligned}
\text { for } & (i=1 ; i<=N ;++i) \\
\text { for } & (j=1 ; j<=i ;++j) \\
& a[i][j]=
\end{aligned}
$$

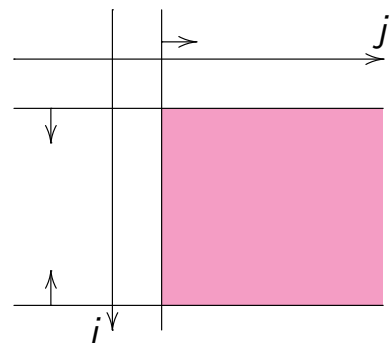

Iteration domain: $P=\{[i, j] \mid i \geq 1 \wedge i \leq N \wedge j \geq 1$

Assumptions on sequential code:

- iterators are integers

- loops with affine bounds

- affine conditions

- affine index expressions 


\section{Polytope Model}

$$
\begin{gathered}
\text { for }(i=1 ; i<=N ;++i) \\
\text { for }(j=1 ; j<=i ;++j) \\
\quad a[i][j]=
\end{gathered}
$$

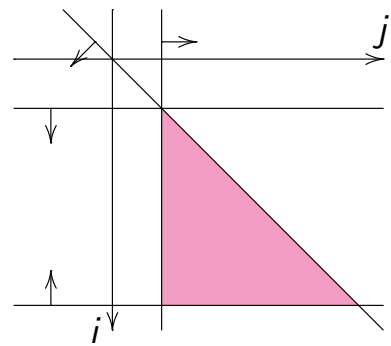

Iteration domain: $P=\{[i, j] \mid i \geq 1 \wedge i \leq N \wedge j \geq 1 \wedge j \leq i\}$

Assumptions on sequential code:

- iterators are integers

- loops with affine bounds

- affine conditions

- affine index expressions 


\section{Polytope Model}

$$
\begin{gathered}
\text { for }(i=1 ; i<=N ;++i) \\
\text { for }(j=1 ; j<=i ;++j) \\
\quad a[i][j]=
\end{gathered}
$$

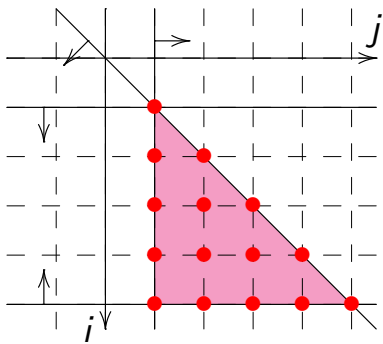

Iteration domain: $P=\{[i, j] \mid i \geq 1 \wedge i \leq N \wedge j \geq 1 \wedge j \leq i\}$

Assumptions on sequential code:

- iterators are integers

- loops with affine bounds

- affine conditions

- affine index expressions 


\section{Lexicographical Order}

\#define $\mathrm{N} 5$

for $(i=1 ; i<=N ;++i)$

for $(j=1 ; j<=i ;++j)$

$a[i][j]=$

$$
S=\{(i, j) \mid i \geq 1 \wedge i \leq N \wedge j \geq 1 \wedge j \leq i\}
$$

Execution order: $(1,1),(2,1),(2,2),(3,1),(3,2),(3,3),(4,1),(4,2),(4,3)$, $(4,4)(5,1),(5,2),(5,3),(5,4),(5,5)$ 


\section{Lexicographical Order}

\#define $\mathrm{N} 5$

for $(i=1 ; i<=N ;++i)$

for $(j=1 ; j<=i ;++j)$

$a[i][j]=$

$$
S=\{(i, j) \mid i \geq 1 \wedge i \leq N \wedge j \geq 1 \wedge j \leq i\}
$$

Execution order: $(1,1),(2,1),(2,2),(3,1),(3,2),(3,3),(4,1),(4,2),(4,3)$, $(4,4)(5,1),(5,2),(5,3),(5,4),(5,5)$

Lexicographical order:

$$
\mathbf{a} \prec \mathbf{b} \equiv \bigvee_{i=1}^{n}\left(a_{i}<b_{i} \wedge \bigwedge_{j=1}^{i-1} a_{j}=b_{j}\right)
$$

$\Rightarrow$ smaller in first position where vectors differ 


\section{Derivation of Polyhedral Process Networks}

Main difficulty: what are the channels and how big are they ?

- dataflow analysis

Given a read from an array element, what was the last write to that array element?

- counting

Given a communication channel, how many tokens are in the channel at any given time?

- computing upper bounds

Given a communication channel, what is the maximal number of tokens in the channel during the execution? 


\section{Derivation of Polyhedral Process Networks}

Main difficulty: what are the channels and how big are they ?

- dataflow analysis

Given a read from an array element, what was the last write to that array element? 


\section{Toy Example}

int $\mathrm{N}$;

int main(void)

\{

int $i, j$;
int $a[N]$;

for $(i=0 ; i<N ;++i)$

$\mathrm{a}[\mathrm{i}]=\operatorname{Read}()$;

for $(i=0 ; i<N ;++i)$

for $(j=0 ; j<N-i ;++j)$

$a[i+j]=f(a[i+j]) ;$

for $(i=0 ; i<N$; ++i)

Write (a[i]);

\} 


\section{Toy Example}

int $\mathrm{N}$;

int main(void)

\{

int $i, j$;
int $a[N]$;

for $(i=0 ; i<N ;++i)$

$\mathrm{a}[\mathrm{i}]=\operatorname{Read}()$;

for $(i=0 ; i<N ;++i)$

for $(j=0 ; j<N-i ;++j)$

$a[i+j]=f(a[i+j])$;

for $(i=0 ; i<N$; ++i)

Write (a[i]);

\} 


\section{Dataflow Analysis}

Given a read from an array element, what was the last write to the same array element before the read?

Step 1: last write through given write access

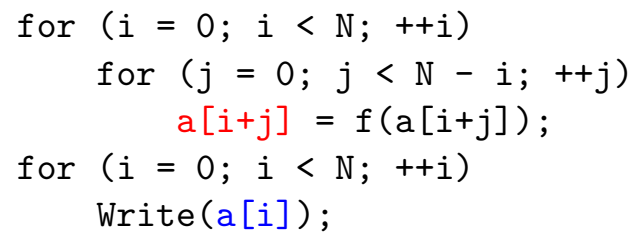




\section{Dataflow Analysis}

Given a read from an array element, what was the last write to the same array element before the read?

Step 1: last write through given write access

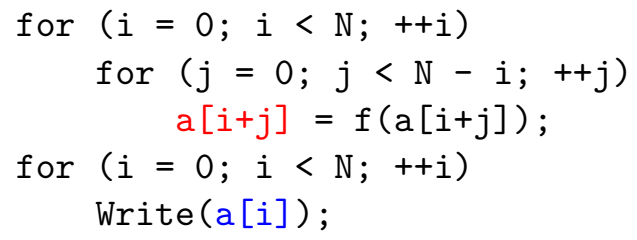




\section{Dataflow Analysis}

Given a read from an array element, what was the last write to the same array element before the read?

Step 1: last write through given write access

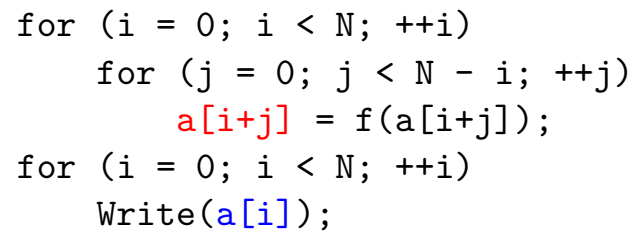

Access relations:

$$
\begin{aligned}
& A_{1}=\{(i, j) \rightarrow(i+j) \mid 0 \leq i<N \wedge 0 \leq j<N-i\} \\
& A_{2}=\{(i) \rightarrow(i) \mid 0 \leq i<N\}
\end{aligned}
$$




\section{Dataflow Analysis}

Given a read from an array element, what was the last write to the same array element before the read?

Step 1: last write through given write access

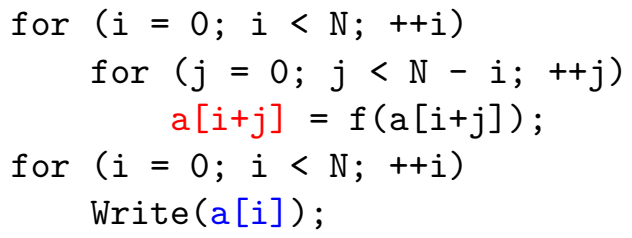

Access relations:

$A_{1}=\{(i, j) \rightarrow(i+j) \mid 0 \leq i<N \wedge 0 \leq j<N-i\}$

$A_{2}=\{(i) \rightarrow(i) \mid 0 \leq i<N\}$

Map to all writes: $R^{\prime}=A_{1}^{-1} \circ A_{2}=\left\{(i) \rightarrow\left(i^{\prime}, i-i^{\prime}\right) \mid 0 \leq i^{\prime} \leq i<N\right\}$ 


\section{Dataflow Analysis}

Given a read from an array element, what was the last write to the same array element before the read?

Step 1: last write through given write access

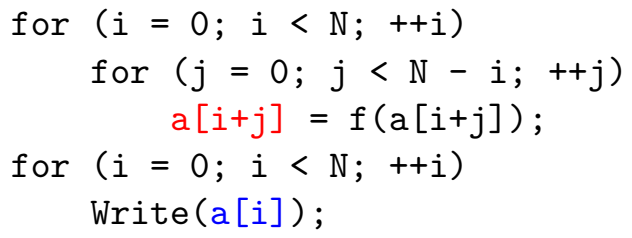

Access relations:

$A_{1}=\{(i, j) \rightarrow(i+j) \mid 0 \leq i<N \wedge 0 \leq j<N-i\}$

$A_{2}=\{(i) \rightarrow(i) \mid 0 \leq i<N\}$

Map to all writes: $R^{\prime}=A_{1}^{-1} \circ A_{2}=\left\{(i) \rightarrow\left(i^{\prime}, i-i^{\prime}\right) \mid 0 \leq i^{\prime} \leq i<N\right\}$

Last write: $R=\operatorname{lexmax} R^{\prime}=\{(i) \rightarrow(i, 0) \mid 0 \leq i<N\}$ 


\section{Dataflow Analysis}

Given a read from an array element, what was the last write to the same array element before the read?

Step 1: last write through given write access

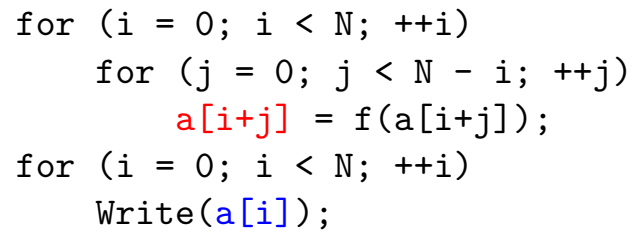

Access relations:

$A_{1}=\{(i, j) \rightarrow(i+j) \mid 0 \leq i<N \wedge 0 \leq j<N-i\}$

$A_{2}=\{(i) \rightarrow(i) \mid 0 \leq i<N\}$

Map to all writes: $R^{\prime}=A_{1}^{-1} \circ A_{2}=\left\{(i) \rightarrow\left(i^{\prime}, i-i^{\prime}\right) \mid 0 \leq i^{\prime} \leq i<N\right\}$ Last write: $R=\operatorname{lexmax} R^{\prime}=\{(i) \rightarrow(i, 0) \mid 0 \leq i<N\}$ In general: impose lexicographical order on shared iterators 


\section{Toy Example}

int $\mathrm{N}$;

int main(void)

\{

int $i, j$;
int $a[N]$;

for $(i=0 ; i<N ;++i)$

$\mathrm{a}[\mathrm{i}]=\operatorname{Read}()$;

for $(i=0 ; i<N ;++i)$

for $(j=0 ; j<N-i ;++j)$

$a[i+j]=f(a[i+j])$;

for $(i=0 ; i<N$; ++i)

Write (a[i]);

\} 


\section{Toy Example}

int $\mathrm{N}$;

int main(void)

\{

int $i, j$;
int $a[N]$;

for $(i=0 ; i<N ;++i)$

$\mathrm{a}[\mathrm{i}]=\operatorname{Read}()$;

for $(i=0 ; i<N ;++i)$

for $(j=0 ; j<N-i ;++j)$

$a[i+j]=f(a[i+j])$;

for $(i=0 ; i<N$; ++i)

Write (a[i]);

\} 


\section{Dataflow Analysis}

Step 1: last write through given write access

Step 2: combination of results over all write accesses 


\section{Dataflow Analysis}

Step 1: last write through given write access

Step 2: combination of results over all write accesses

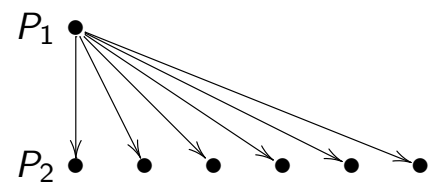




\section{Dataflow Analysis}

Step 1: last write through given write access

Step 2: combination of results over all write accesses + read accesses from same statement/process

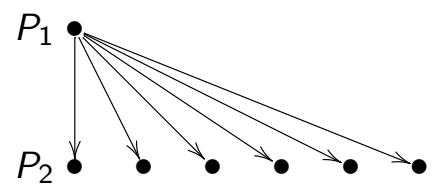




\section{Dataflow Analysis}

Step 1: last write through given write access

Step 2: combination of results over all write accesses + read accesses from same statement/process
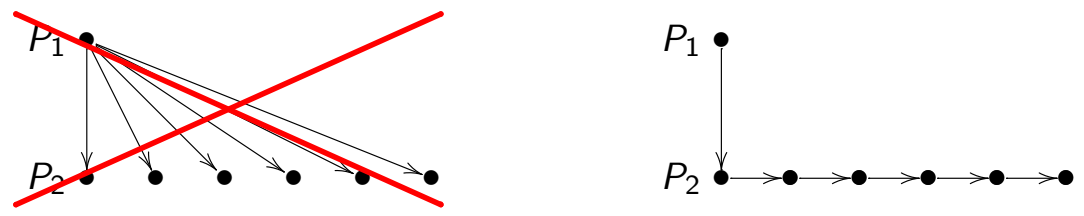


\section{Dataflow Analysis: Result on Toy Example}

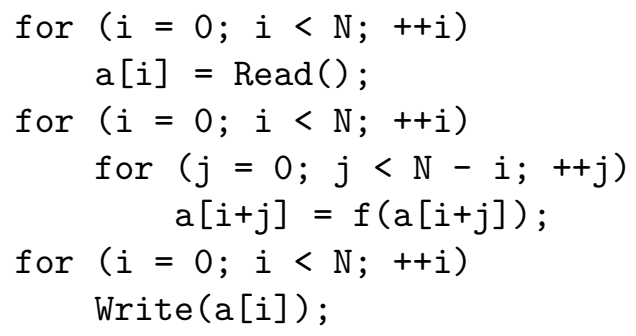

Write 


\section{FIFO or Reordering Channel?}

FIFOs are more efficient to implement in hardware

$\Rightarrow$ check whether communication channel is a FIFO

FIFO: reads occur in same order as corresponding writes 


\section{FIFO or Reordering Channel?}

FIFOs are more efficient to implement in hardware

$\Rightarrow$ check whether communication channel is a FIFO

FIFO: reads occur in same order as corresponding writes

Communication channel with dependence relation $R$ is not a FIFO iff there is a pair of reads $\mathbf{r}_{1}$ and $\mathbf{r}_{2}$ such that $\mathbf{r}_{1}$ occurs before $\mathbf{r}_{2}$, but the writes occur in the opposite order

$$
T=\left\{\left(\mathbf{r}_{1}, \mathbf{r}_{2}\right) \in(\operatorname{dom} R)^{2} \mid \mathbf{r}_{1} \prec \mathbf{r}_{2} \wedge R\left(\mathbf{r}_{1}\right) \succ R\left(\mathbf{r}_{2}\right)\right\}
$$

$T=\emptyset \Rightarrow$ communication channel is FIFO 


\section{FIFO or Reordering Channel?}

FIFOs are more efficient to implement in hardware

$\Rightarrow$ check whether communication channel is a FIFO

FIFO: reads occur in same order as corresponding writes

Communication channel with dependence relation $R$ is not a FIFO iff there is a pair of reads $\mathbf{r}_{1}$ and $\mathbf{r}_{2}$ such that $\mathbf{r}_{1}$ occurs before $\mathbf{r}_{2}$, but the writes occur in the opposite order

$$
T=\left\{\left(\mathbf{r}_{1}, \mathbf{r}_{2}\right) \in(\operatorname{dom} R)^{2} \mid \mathbf{r}_{1} \prec \mathbf{r}_{2} \wedge R\left(\mathbf{r}_{1}\right) \succ R\left(\mathbf{r}_{2}\right)\right\}
$$

$T=\emptyset \Rightarrow$ communication channel is FIFO

From now on we assume all channels are FIFOs (Reordering channels require extra reordering buffer) 


\section{Derivation of Polyhedral Process Networks}

Main difficulty: what are the channels and how big are they ?

- dataflow analysis

Given a read from an array element, what was the last write to that array element? 


\section{Derivation of Polyhedral Process Networks}

Main difficulty: what are the channels and how big are they ?

- dataflow analysis

Given a read from an array element, what was the last write to that array element?

Lexicographic maximum of parametric polytope (isl, piplib, PPL)

$\Rightarrow$ piecewise quasi-affine map 


\section{Commercial Break: isl}

isl is an

- LGPL

- thread-safe

- C

library for manipulating integer sets and relations

- bounded by affine constraints

- involving parameters and

- existentially quantified variables

Supported operations include

- basic operations such as intersection, union and set difference

- integer projection

- set coalescing

- convex hull

- integer affine hull

- computing the lexicographic minimum of a relation, 


\section{Derivation of Polyhedral Process Networks}

Main difficulty: what are the channels and how big are they?

- dataflow analysis

Given a read from an array element, what was the last write to that array element?

Lexicographic maximum of parametric polytope (isl, piplib, PPL)

$\Rightarrow$ piecewise quasi-affine map 


\section{Derivation of Polyhedral Process Networks}

Main difficulty: what are the channels and how big are they?

- dataflow analysis

Given a read from an array element, what was the last write to that array element?

Lexicographic maximum of parametric polytope (isl, piplib, PPL)

$\Rightarrow$ piecewise quasi-affine map

- counting

Given a communication channel, how many tokens are in the channel at any given time? 


\section{Parametric Counting}

Given a communication channel, how many tokens are in the channel at any given time?

Consider intra-process communication channel (FIFO)

Iteration domain of process: $S$

Dependence relation of channel: $R \subset S \times S$ 


\section{Parametric Counting}

Given a communication channel, how many tokens are in the channel at any given time?

Consider intra-process communication channel (FIFO)

Iteration domain of process: $S$

Dependence relation of channel: $R \subset S \times S$

Number of tokens in channel before iteration $\mathbf{i} \in S$ : number of writes to channel before $\mathbf{i}$ minus number of reads from channel before $\mathbf{i}$

$$
n(\mathbf{i})=\#\{\mathbf{w} \in \operatorname{ran} R \mid \mathbf{w} \prec \mathbf{i}\}-\#\{\mathbf{r} \in \operatorname{dom} R \mid \mathbf{w} \prec \mathbf{i}\}
$$




\section{Parametric Counting Example}

Number of tokens in channel before iteration $\mathbf{i} \in S$ : number of writes to channel before $\mathbf{i}$ minus number of reads from channel before $\mathbf{i}$

$$
n(\mathbf{i})=\#\{\mathbf{w} \in \operatorname{ran} R \mid \mathbf{w} \prec \mathbf{i}\}-\#\{\mathbf{r} \in \operatorname{dom} R \mid \mathbf{w} \prec \mathbf{i}\}
$$

for $(i=0 ; i<N ;++i)$

$$
\begin{gathered}
\text { for }(j=0 ; j<N-i ;++j) \\
a[i+j]=f(a[i+j]) ;
\end{gathered}
$$

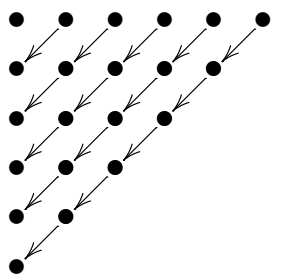

$$
\begin{aligned}
& R=\{(i, j) \rightarrow(i-1, j+1) \mid 1 \leq i \wedge 0 \leq j<N-i\} \\
& n(i, j)= \begin{cases}j-1 & \text { if } i=0 \wedge 1 \leq j<N \\
N-i & \text { if } j=0 \wedge 1 \leq i<N \\
N-i-1 & \text { if } 1 \leq i<N \wedge 1 \leq j<N-i\end{cases}
\end{aligned}
$$




\section{Parametric Counting}

Number of tokens in channel before iteration $\mathbf{i} \in S$ : number of writes to channel before $\mathbf{i}$ minus number of reads from channel before $\mathbf{i}$

$$
n(\mathbf{i})=\#\{\mathbf{w} \in \operatorname{ran} R \mid \mathbf{w} \prec \mathbf{i}\}-\#\{\mathbf{r} \in \operatorname{dom} R \mid \mathbf{w} \prec \mathbf{i}\}
$$

Ordering of iterations

- fixed within the same process

- unknown at compile-time between different processes

$\Rightarrow$ combine all processes into a single process

$\Rightarrow$ fix a particular schedule

$\Rightarrow$ perform counting in the combined process

Fixed schedule only used during buffer size computation

Computed sizes will be valid for given schedule

$\Rightarrow$ sizes allow for deadlock-free execution (at least one valid schedule) 


\section{Process Merging}

Given: iteration domains of dimension $d_{i} \leq D$

(2) Place in a common iteration space of dimension $D$

(no change of order within an iteration domain) Heuristic: identify dimensions related through dependence relation 


\section{Process Merging: Common Iteration Space}

$$
\begin{aligned}
& \text { for }(i=0 ; i<N ;++i) \\
& \quad \text { for }(j=0 ; j<N-i ;++j)
\end{aligned}
$$$$
a[i+j]=f(a[i+j]) \text {; }
$$

for $(i=0 ; i<N ;++i)$

Write (a $[i])$;

$$
R=\left\{(i) \rightarrow\left(i^{\prime}, j^{\prime}\right) \mid 0 \leq i<N \wedge i^{\prime}=i \wedge j^{\prime}=0\right\}
$$




\section{Process Merging: Common Iteration Space}

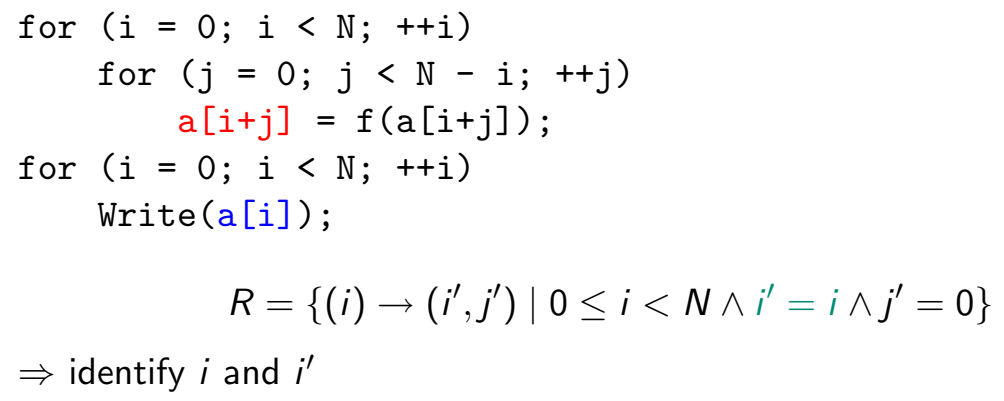




\section{Process Merging: Common Iteration Space}

$$
\begin{aligned}
& \text { for }(i=0 ; i<N ;++i) \\
& \quad \text { for }(j=0 ; j<N-i ;++j)
\end{aligned}
$$$$
a[i+j]=f(a[i+j]) \text {; }
$$

for $(i=0 ; i<N ;++i)$

Write (a $[i])$;

$$
R=\left\{(i) \rightarrow\left(i^{\prime}, j^{\prime}\right) \mid 0 \leq i<N \wedge i^{\prime}=i \wedge j^{\prime}=0\right\}
$$

$\Rightarrow$ identify $i$ and $i^{\prime}$
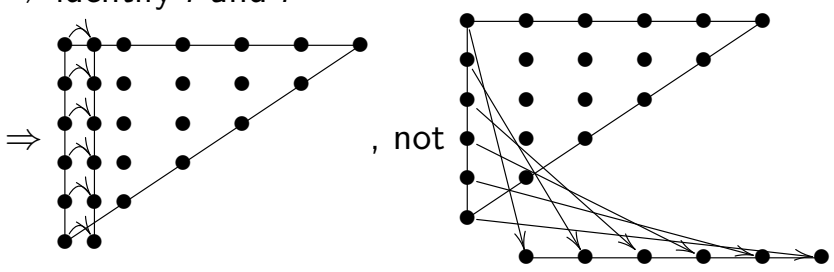


\section{Process Merging}

Given: iteration domains of dimension $d_{i} \leq D$

(1) Place in a common iteration space of dimension $D$

(no change of order within an iteration domain) Heuristic: identify dimensions related through dependence relation 


\section{Process Merging}

Given: iteration domains of dimension $d_{i} \leq D$

( $)$ Place in a common iteration space of dimension $D$

(no change of order within an iteration domain) Heuristic: identify dimensions related through dependence relation

(2) Add constant offsets to all iteration domains (without violating any dependence) Heuristic: put dependent iterations "close" together 


\section{Derivation of Polyhedral Process Networks}

Main difficulty: what are the channels and how big are they?

- dataflow analysis

Lexicographic maximum of parametric polytope (isl, piplib, PPL)

$\Rightarrow$ piecewise quasi-affine map

- counting

Given a communication channel, how many tokens are in the channel at any given time? 


\section{Derivation of Polyhedral Process Networks}

Main difficulty: what are the channels and how big are they?

- dataflow analysis

Lexicographic maximum of parametric polytope (isl, piplib, PPL)

$\Rightarrow$ piecewise quasi-affine map

- counting

Given a communication channel, how many tokens are in the channel at any given time?

Number of elements in parametric polytope (barvinok)

$\Rightarrow$ piecewise quasi-polynomial 


\section{Derivation of Polyhedral Process Networks}

Main difficulty: what are the channels and how big are they?

- dataflow analysis

Lexicographic maximum of parametric polytope (isl, piplib, PPL)

$\Rightarrow$ piecewise quasi-affine map

- counting

Given a communication channel, how many tokens are in the channel at any given time?

Number of elements in parametric polytope (barvinok)

$\Rightarrow$ piecewise quasi-polynomial

- computing upper bounds

Given a communication channel, what is the maximal number of tokens in the channel during the execution? 


\section{Computing Parametric Upper Bounds}

Given a communication channel, what is the maximal number of tokens in the channel during the execution?

$$
\begin{gathered}
\text { for }(i=0 ; i<N ;++i) \\
\text { for }(j=0 ; j<N-i ;++j) \\
\quad a[i+j]=f(a[i+j]) ;
\end{gathered}
$$

Number of tokens in FIFO at $(i, j)$ :

$$
n(i, j)= \begin{cases}j-1 & \text { if } i=0 \wedge 1 \leq j<N \\ N-i & \text { if } j=0 \wedge 1 \leq i<N \\ N-i-1 & \text { if } 1 \leq i<N \wedge 1 \leq j<N-i\end{cases}
$$




\section{Computing Parametric Upper Bounds}

Given a communication channel, what is the maximal number of tokens in the channel during the execution?

$$
\begin{gathered}
\text { for }(i=0 ; i<N ;++i) \\
\text { for }(j=0 ; j<N-i ;++j) \\
\quad a[i+j]=f(a[i+j]) ;
\end{gathered}
$$

Number of tokens in FIFO at $(i, j)$ :

$$
n(i, j)= \begin{cases}j-1 & \text { if } i=0 \wedge 1 \leq j<N \\ N-i & \text { if } j=0 \wedge 1 \leq i<N \\ N-i-1 & \text { if } 1 \leq i<N \wedge 1 \leq j<N-i\end{cases}
$$

Maximal number of tokens:

$$
\max \left(\max _{1 \leq j<N} j-1, \max _{1 \leq i<N} N-i, \max _{1 \leq i<N \wedge 1 \leq j<N-i} N-i-1\right)
$$




\section{Computing Parametric Upper Bounds}

Maximal number of tokens:

$$
\max \left(\max _{1 \leq j<N} j-1, \max _{1 \leq i<N} N-i, \max _{1 \leq i<N \wedge 1 \leq j<N-i} N-i-1\right)
$$

$\Rightarrow$ can be approximated using Bernstein expansion

- Ideally:

- maximum over

- integer points

- Bernstein expansion gives

- upper bound over

- rational points

In practice very accurate 


\section{Derivation of Polyhedral Process Networks}

Main difficulty: what are the channels and how big are they?

- dataflow analysis

Lexicographic maximum of parametric polytope (isl, piplib, PPL)

$\Rightarrow$ piecewise quasi-affine map

- counting

Number of elements in parametric polytope (barvinok)

$\Rightarrow$ piecewise quasi-polynomial

- computing upper bounds

Given a communication channel, what is the maximal number of tokens in the channel during the execution? 


\section{Derivation of Polyhedral Process Networks}

Main difficulty: what are the channels and how big are they?

- dataflow analysis

Lexicographic maximum of parametric polytope (isl, piplib, PPL)

$\Rightarrow$ piecewise quasi-affine map

- counting

Number of elements in parametric polytope (barvinok)

$\Rightarrow$ piecewise quasi-polynomial

- computing upper bounds

Given a communication channel, what is the maximal number of tokens in the channel during the execution?

Upper bound of parametric polynomial over parametric polytope (bernstein) 


\section{Outline}

(1) Introduction and Motivation

(2) Polyhedral Process Networks

- Polytope Model

- Dataflow Analysis

- Parametric Counting

- Computing Parametric Upper Bounds

(3) Conclusion 


\section{Conclusion}

Derivation of Polyhedral Process Networks requires

- Parametric Integer Programming (lexmax)

$\Rightarrow$ available in isl, piplib and PPL (soon)

- Enumeration of parametric polytopes

$\Rightarrow$ available in barvinok

- Bounds on polynomial over parametric polytopes

$\Rightarrow$ available in bernstein

Prototype implementation available from

http://www.kotnet.org/ skimo/loop/isa-0.09.tar.bz2 


\section{Conclusion}

Derivation of Polyhedral Process Networks requires

- Parametric Integer Programming (lexmax)

$\Rightarrow$ available in isl, piplib and PPL (soon)

- Enumeration of parametric polytopes

$\Rightarrow$ available in barvinok

- Bounds on polynomial over parametric polytopes

$\Rightarrow$ available in bernstein

Prototype implementation available from

http://www.kotnet.org/ skimo/loop/isa-0.09.tar.bz2

bernstein library included in barvinok, available from http://freshmeat.net/projects/barvinok/

isl available from http://freshmeat.net/projects/isl/ 